\title{
Inventory Control by Multiple Service Levels under Unreliable Supplying Condition
}

\author{
Byungsoo $\mathrm{Na},{ }^{1}$ Jinpyo Lee, ${ }^{2}$ and Hyung Jun $\mathrm{Ahn}^{2}$ \\ ${ }^{1}$ Division of Business Administration, Korea University, Sejong, Republic of Korea \\ ${ }^{2}$ College of Business Administration, Hongik University, Seoul, Republic of Korea \\ Correspondence should be addressed to Jinpyo Lee; jinpyo.lee@hongik.ac.kr
}

Received 28 March 2016; Accepted 31 July 2016

Academic Editor: Francisco Gordillo

Copyright (c) 2016 Byungsoo Na et al. This is an open access article distributed under the Creative Commons Attribution License, which permits unrestricted use, distribution, and reproduction in any medium, provided the original work is properly cited.

\begin{abstract}
We consider an inventory system where there is random demand from customers as well as unreliable supplying capacity from supplier. In many real-world cases, supplier might fail to satisfy the amount of order from retailers or producers so that only partial proportion of order is satisfied or even fail to deliver all of the order. Moreover, recently a concern regarding unreliable supplying capacity has been increasing since the globalization makes the retailer or producer face the extended supply network with complicated and risky supplying capacity. Also, we consider two classified customers, of which one is willing to pay extra charge for expedited delivery service but the other is not reluctant to delay the delivery without any extra charge. We show that there exists an optimal threshold for inventory and price for each service level in the following sense: if the inventory level is less than the predetermined threshold, then the retailer or producer needs to order up to the threshold level and offer threshold price corresponding to service level. Otherwise, the retailer does not need to order. The risk of stockout due to unreliable supplying capacity can be mitigated by the dynamic pricing and inventory control with multiple service levels.
\end{abstract}

\section{Introduction}

Coordination of dynamic inventory and pricing control has been used as main strategy for many companies such as Amazon, Dell, and J. C. Penny [1]. In many traditional works for inventory control problem, how to maximize the profit and control the uncertainty of demand from customers has been a stark issue, in which the optimal policy has been provided using either ordering quantity or pricing of each product. Even some works show the optimal inventory control policy using multiple pricing on single product depending on the service levels. However, in addition to the random demand from customers, it is not well addressed that most firms experience the unreliable supplying capacity from supplier, which is another randomness carried from supplier. For example, suppose that Amazon.com orders 100 books to a publisher. However, the publisher might fail to fulfill the order and it may deliver only some of the ordered books (i.e., 80 books) for its own supplying capacity problem. Some recent works address this unreliable supply issue which is incorporated with pricing on the product and replenishment decisions but only addresses the pricing policy on a product with a single-service level [2]. However, as shown in many online stores, each product is delivered with more than a single-service level where faster service can be suggested to the customer willing to pay an extra charge. So, how to balance the demand and supply by incorporating multiple pricing corresponding to the service level is an important issue faced by many firms, especially online firms. In this paper, we show that there can be an optimal policy to an inventory control problem in which each product is priced depending on the service level and the supplying capacity from the supplier is also random in addition to the randomness of demand from the customer.

\section{Literatures Review}

Two streams of literatures are related to this paper:

(1) The inventory/production or pricing control problem under reliable supplying capacity. 
(2) The inventory/production or pricing control problem under unreliable supplying capacity.

There are many literatures that study the inventory or pricing control problem under reliable supplying capacity. Reference [1] gives a comprehensive survey on this problem, in which only a single-service level is considered. References $[3,4]$ address a single-period problem under risk neutrality, which is addressed using Newsvendor Model. Reference [5] suggests optimal replenishment and pricing policy of a single-period model under risk aversion. For multiperiod system, when total ordering cost is a linear function of ordering quantity, a base stock list price is shown to be optimal for single-service level in [6] and for multiple service level in [7]. References [8, 9] show that, for multiperiod system, there is an optimal inventory and pricing policy when a fixed ordering cost is considered and backlogging is allowed. Reference [10] provides an optimal inventory and pricing policy for multiperiod system when a fixed ordering cost is considered and lost-sales are allowed. Reference [11] addresses joint inventory and pricing model where there is a single item with stochastic demand subject to reference effects and the random demand is a function of the current price and the reference price is acting as a benchmark with which customers compare the current price. Reference [12] suggests the joint pricing and inventory model for a stochastic inventory system with perishable products, where the inventory system under random demand and reliable supplying capacity is modeled by a continuous-time stochastic differential equation. Reference [13] considers joint pricing and inventory replenishment decision problem over an infinite horizon where sequentially arriving customers are forward-looking to the price of a product sold by a seller. In their model, so-called strategic customers wait and monitor prices offered from the seller and then anticipate a lower future price. Reference [14] addresses a joint pricing and production decision problem for perishable items sold to price-sensitive customers, assuming that shortages are not allowed.

The other stream of literature relevant to our paper is the inventory/production or pricing control problem under unreliable supplying capacity. Reference [15] surveys the literature on how to quantitatively determine lot sizes when production or procurement yields are uncertain. Reference [16] addresses an inventory control model for a periodic review with unreliable production capacity, random yields, and uncertain demand but does not consider the dynamic pricing by considering the price as exogenous and thus minimizing the total discounted expected costs which are production, holding, and shortage costs. References [17, 18] address the joint inventory and pricing decision problem with random demand and unreliable suppling capacity, in which there are no multiple service levels. Reference [19] addresses the inventory (but no pricing) decision model for production-inventory systems in which the stock can deteriorate with time and supplying capacity from multiple suppliers is unreliable. Reference [20] investigates an optimal inventory strategy for a risk-averse retailer facing demand uncertainty and unreliable supply in which the price is given and is exogenous. Reference [21] considers a risk-neutral monopolist manufacturer ordering a key component from several suppliers using a single-period model, in which some suppliers might face risks of complete supplying disruptions, which are called unreliable suppliers. Reference [22] suggests optimal sourcing strategies without pricing decision when there are a perfectly reliable supplier and an unreliable supplier.

\section{Assumptions and Notations}

In this paper, the following notations are used:

(1) $c$ : per unit marginal cost,

(2) $p_{R, t}:$ price charged for regular service in period $t$,

(3) $p_{E, t}:$ price charged for express service in period $t$,

(4) $p_{t}=p_{E, t}-p_{R, t}$ : extra charge for the express service in period $t$ on $[0, \bar{p}]$,

(5) $\epsilon_{t}$ : random uncertainty term of demand which had a known distribution,

(6) $D_{E, t}\left(p_{R, t}, p_{E, t}\right)$ : demand for the express service,

(7) $D_{R, t}\left(p_{R, t}\right)$ : demand for the regular service,

(8) $x_{t}$ : inventory level at the beginning of each period $t$ before ordering,

(9) $y_{t}$ : inventory level at the beginning of each period $t$ after ordering,

(10) $h_{t}(I)$ : inventory cost (holding or backlogging) at the end of each period $t$.

Assumption 1. Backlogging is allowed.

Assumption 2. Replenishment after ordering becomes available instantaneously.

Traditionally, in operations research literatures, the customer's demand is assumed to be decreasing concave function or simply a decreasing linear function with the price as an input variable. Also, the expected demand value is assumed to be finite and strictly decreasing as the price increases, which is generally acceptable and reasonable. This decreasing property of demand can be negative if the value of price is sufficiently large. Thus, we need to make another assumption such that a feasible price should be selected on the restricted range in order to be nonnegative valued $[6,9,23,24]$.

Assumption 3. In each period $t=1,2, \ldots, T$, the demand function for regular service $D_{R, t}\left(p_{R, t}, p_{E, t}\right)=d_{R, t}\left(p_{t}\right)$, where $d_{R, t}\left(p_{t}\right)$ is given by $d_{R, t}\left(p_{E, t}-p_{R, t}\right)$, and nondecreasing linear function in $p_{t}\left(=p_{E, t}-p_{R, t}\right) \in[0, \bar{p}]$. The demand function for express service $D_{E, t}\left(p_{R, t}, p_{E, t}\right)=d_{E, t}\left(p_{R, t}, p_{t}\right)$, where $d_{E, t}\left(p_{R, t}, p_{t}\right)$ is given by $a\left(p_{R, t}, \epsilon_{t}\right)-D_{R, t}\left(p_{R, t}, p_{E, t}\right)=$ $a\left(p_{R, t}, \epsilon_{t}\right)-d_{R, t}\left(p_{t}\right)$, which is nonincreasing linear function in $p_{R, t} \in\left[\underline{p}_{R}, \bar{p}_{R}\right]$ and $p_{t} \in[0, \bar{p}]$. Moreover, $a\left(p_{R, t}, \epsilon_{t}\right)$ is the possible maximum demand and nonincreasing and linear function of $p_{R, t} \in\left[c, \bar{p}_{R}\right]$. Moreover, values for $\bar{p}$ and $\bar{p}_{R}$ are taken such that $a\left(\bar{p}_{R}, \epsilon_{t}\right)-D_{R, t}\left(p_{R, t}, p_{E, t}\right)$ is nonnegative with probability 1 . 
Assumption 3 came up from the following insight. The number of customers using the express service would decrease as the price difference $p_{t}$ between regular and express service increases. For example, when you try to buy some product from an online store, the higher the price difference between regular shipping and express shipping service are, the more you are reluctant to select the express shipping service. Thus, as the price difference $p_{t}$ between regular and express service increases, the customers who are reluctant to select express service will become the customers for regular service.

Assumption 4. The revenue in each period $t=1,2, \ldots, T$ is given by

$$
\begin{gathered}
p_{R, t} E\left[D_{R, t}\left(p_{R, t}, p_{E, t}\right)\right]+p_{E, t} E\left[D_{E, t}\left(p_{R, t}, p_{E, t}\right)\right] \\
=p_{R, t} E\left[d_{R, t}\left(p_{t}\right)\right]+p_{E, t} E\left[d_{E, t}\left(p_{R, t}, p_{t}\right)\right]
\end{gathered}
$$

and is finite and concave for $p_{R, t} \in\left[\underline{p}_{R}, \bar{p}_{R}\right]$ and $p_{t} \in[0, \bar{p}]$, where $p_{t}=p_{E, t}-p_{R, t}$.

Assumption 5. $H_{t}(I)=E\left[h_{t}(I)\right]$ is convex in $I$ and $H_{t}(0)=0$ in each $t=1, \ldots, T$.

Assumption 6. $\lim _{y \rightarrow \pm \infty} H_{t}\left(y-D_{E, t}\left(p_{R, t}, p_{E, t}\right)\right)=$ $\lim _{y \rightarrow \pm \infty}\left(c y+H_{t}\left(y-D_{E, t}\left(p_{R, t}, p_{E, t}\right)\right)\right)=\infty$ for any $p_{R, t} \in\left[\underline{p}_{R}\right.$, $\left.\bar{p}_{R}\right]$ and $p_{t} \in[0, \bar{p}]$.

\section{Mathematical Formulation}

We consider an additive demand model, where the demand uncertainty for regular and express service is represented by an additive random noise $\epsilon_{t}$, respectively,

$$
\begin{aligned}
& D_{R, t}\left(p_{R, t}, p_{E, t}\right)=d_{R, t}\left(p_{t}\right)=\mathscr{D}_{R, t}\left(p_{t}\right)+\epsilon_{t}, \\
& D_{E, t}\left(p_{R, t}, p_{E, t}\right)=d_{E, t}\left(p_{R, t}, p_{t}\right)=\mathscr{D}_{E, t}\left(p_{R, t}, p_{t}\right)+\epsilon_{t} .
\end{aligned}
$$

We assume that $\epsilon_{t}$ has mean zero and support $[\underline{\epsilon}, \bar{\epsilon}]$. For any feasible choice of $p_{R, t} \in\left[\underline{p}_{R}, \bar{p}_{R}\right]$ and $p_{E, t} \in\left[\underline{p}_{E}, \bar{p}_{E}\right]$, the demand is positive with probability one and the average demands $E\left[D_{R, t}\left(p_{R, t}, p_{E, t}\right)\right]$ and $E\left[D_{E, t}\left(p_{R, t}, p_{E, t}\right)\right]$ are finite.

Given the inventory level $I_{t}$ in period $t$, the inventory level $I_{t+1}$ in period $t+1$ is given as follows:

$$
I_{t+1}=I_{t}+\left(y_{t}-I_{t}\right) \wedge k-d_{E, t}\left(p_{R, t}, p_{t}\right),
$$

where $k \in\left[0, y_{t}-I_{t}\right]$ in period $t$ and $a \wedge b=\min [a, b]$. Let $g_{t}(I, x)$ be the optimal profit function in period $t$ when the inventory level is $I$ and the demand from period $t-1$ is $x$. Then, the optimality equation is given by

$$
g_{t}(I, x)=\max _{y \geq I, \underline{p}_{R} \leq p_{R} \leq \bar{p}_{R}, \underline{p} \leq p \leq \bar{p}} \pi_{t}\left(I ; y, p_{R}, p\right),
$$

where

$$
\begin{aligned}
& \pi_{t}\left(I ; y, p_{R}, p\right) \\
&=p_{R} E\left[d_{R}(p)\right]+p_{E} E\left[d_{E}\left(p_{R}, p\right)\right] \\
&-c E[(I+k) \wedge y-I] \\
&-E\left[H\left((I+k) \wedge y-d_{E}\left(p_{R}, p\right)\right)\right] \\
&+\alpha E\left[g_{t+1}\left((I+k) \wedge y-d_{E}\left(p_{R}, p\right), d_{R}(p)\right)\right] .
\end{aligned}
$$

From Lemma 7, we can expect a dynamic programming model with single state as an input variable which is equivalent to (4). Moreover, we can see that the optimal solution to the equivalent dynamic programming model with single state can be translated into the optimal solution to (4).

Lemma 7. Let $\bar{I}$ and $\bar{y}$ be defined as $I-x$ and $y-x$, respectively. Then, $\left(y_{t}^{*}+x, p_{R, t}^{*}, p_{t}^{*}\right)$ is the set of optimal solutions to (4) if and only if $\left(y_{t}^{*}, p_{R, t}^{*}, p_{t}^{*}\right)$ is the set of optimal solutions to

$$
\mathrm{G}_{t}(\bar{I})=\max _{\bar{y} \geq \bar{I} \underline{\underline{p}}_{R} \leq p_{R} \leq \bar{p}_{R}, \underline{p} \leq p \leq \bar{p}} \Pi_{t}\left(\bar{I}: \bar{y}, p_{R}, p\right),
$$

where

$$
\begin{aligned}
\Pi_{t}(\bar{I} & \left.: \bar{y}, p_{R}, p\right) \\
= & p_{R} E\left[d_{R}(p)\right]+p_{E} E\left[d_{E}\left(p_{R}, p\right)\right] \\
& -c E[(\bar{I}+k) \wedge \bar{y}-\bar{I}] \\
& -E\left[H\left((\bar{I}+k) \wedge \bar{y}-d_{E}\left(p_{R}, p\right)\right)\right] \\
& +\alpha E\left[\mathrm{G}_{t+1}\left((\bar{I}+k) \wedge \bar{y}-d_{E}\left(p_{R}, p\right)-d_{R}(p)\right)\right] .
\end{aligned}
$$

Proof. It is enough to show that, for all period $t$,

$$
\pi_{t}\left(I ; y, p_{R}, p\right)=\Pi_{t}\left(\bar{I}: \bar{y}, p_{R}, p\right) \quad \forall I, x, y, p_{R}, p .
$$

Since

$$
\begin{aligned}
(\bar{I}+k) \wedge \bar{y} & =(I-x+k) \wedge(y-x) \\
& = \begin{cases}I-x+k, & \text { if } I-x+k \leq y-x \\
y-x, & \text { otherwise }\end{cases} \\
& =(I+k) \wedge y-x,
\end{aligned}
$$

for all period $t$, the first four terms of $\pi_{t}\left(I ; y, p_{R}, p\right)$ and $\Pi_{t}(\bar{I}$ : $\left.\bar{y}, p_{R}, p\right)$ are equal to each other as follows:

$$
\begin{aligned}
p_{R} E[ & \left.d_{R}(p)\right]+p_{E} E\left[d_{E}\left(p_{R}, p\right)\right] \\
& -c E[(\bar{I}+k) \wedge \bar{y}-\bar{I}] \\
& -E\left[H\left((\bar{I}+k) \wedge \bar{y}-d_{E}\left(p_{R}, p\right)\right)\right] \\
= & p_{R} E\left[d_{R}(p)\right]+p_{E} E\left[d_{E}\left(p_{R}, p\right)\right] \\
& -c E[(I+k) \wedge y-I] \\
& -E\left[H\left((I+k) \wedge y-d_{E}\left(p_{R}, p\right)-x\right)\right] .
\end{aligned}
$$


In period $T$, trivially $g_{T}(I, x)=\mathrm{G}_{T}(\bar{I})=0 . \operatorname{In}$ period $T-1$,

$$
\begin{aligned}
\pi_{T-1} & \left(I ; y, p_{R}, p\right) \\
= & p_{R} E\left[d_{R, T-1}(p)\right]+p_{E} E\left[d_{E}\left(p_{R, T-1}, p\right)\right] \\
& -c E[(I+k) \wedge y-I] \\
& -E\left[H\left((I+k) \wedge y-d_{E, T-1}\left(p_{R}, p\right)-x\right)\right] \\
= & p_{R} E\left[d_{R, T-1}(p)\right]+p_{E} E\left[d_{E, T-1}\left(p_{R}, p\right)\right] \\
& -c E[(\bar{I}+k) \wedge \bar{y}-\bar{I}] \\
& -E\left[H\left((\bar{I}+k) \wedge \bar{y}-d_{E, T-1}\left(p_{R}, p\right)\right)\right] \\
= & \Pi_{T-1}\left(\bar{I}: \bar{y}, p_{R}, p\right) .
\end{aligned}
$$

So, $g_{T-1}(I, x)=\mathrm{G}_{T-1}(\bar{I})$ with $\bar{I}=I-x$. By induction, suppose that, for any $I, x$ with $\bar{I}=I-x$,

$$
g_{n}(I, x)=\mathrm{G}_{n}(\bar{I}), \quad n=T, T-1, \ldots, t+1 .
$$

Then

$$
\begin{aligned}
\pi_{t}\left(I ; y, p_{R}, p\right) \\
=p_{R} E\left[d_{R}(p)\right]+p_{E} E\left[d_{E}\left(p_{R}, p\right)\right] \\
\quad-c E[(I+k) \wedge y-I] \\
\quad-E\left[H\left((I+k) \wedge y-d_{E}\left(p_{R}, p\right)-x\right)\right] \\
\quad+\alpha E\left[g_{t+1}\left((I+k) \wedge y-d_{E}\left(p_{R}, p\right)-x, d_{R}(p)\right)\right] \\
=p_{R} E\left[d_{R}(p)\right]+p_{E} E\left[d_{E}\left(p_{R}, p\right)\right] \\
\quad-c E[(\bar{I}+k) \wedge \bar{y}-\bar{I}] \\
\quad-E\left[H\left((\bar{I}+k) \wedge \bar{y}-d_{E}\left(p_{R}, p\right)\right)\right] \\
\quad+\alpha E\left[G_{t+1}\left((\bar{I}+k) \wedge \bar{y}-d_{E}\left(p_{R}, p\right)-d_{R}(p)\right)\right] .
\end{aligned}
$$

The second equality holds since $(\bar{I}+k) \wedge \bar{y}-d_{E}\left(p_{R}, p\right)-d_{R}(p)=$ $(I+k) \wedge y-d_{E}\left(p_{R}, p\right)-x-d_{R}(p)$. Therefore, the result holds.

4.1. Optimal Inventory Control Policy. Now, we will show the following optimal inventory control policy: if the inventory level at each period $t$ before ordering is less than a predetermined level, then we need to order such that the inventory level is increased up to the predetermined level. Otherwise, no ordering will be made.

Lemma 8. Suppose that $\mathrm{G}_{t+1}(\cdot)$ is concave function and, for given $p_{R}$ and $p$, let $\bar{y}^{*}\left(p_{R}, p\right)$ be the maximizer of

$$
\begin{aligned}
& p_{R} d_{R}(p)+p_{E} d_{E}\left(p_{R}, p\right)-c(\bar{y}-I) \\
& -H\left(\bar{y}-d_{E}\left(p_{R}, p\right)\right) \\
& +\alpha \mathrm{G}_{t+1}\left(\bar{y}-d_{E}\left(p_{R}, p\right)-d_{R}(p)\right),
\end{aligned}
$$

where $p_{E}=p_{R}+p$. Then, $\bar{y}^{*}\left(p_{R}, p\right)$ is also the maximizer of

$$
\begin{aligned}
p_{R} E & {\left[d_{R}(p)\right]+p_{E} E\left[d_{E}\left(p_{R}, p\right)\right] } \\
- & c E[(\bar{I}+k) \wedge \bar{y}-\bar{I}] \\
& -E\left[H\left((\bar{I}+k) \wedge \bar{y}-d_{E}\left(p_{R}, p\right)\right)\right] \\
& +\alpha E\left[G_{t+1}\left((\bar{I}+k) \wedge \bar{y}-d_{E}\left(p_{R}, p\right)-d_{R}(p)\right)\right]
\end{aligned}
$$

for any $\bar{I}$ and, moreover,

$$
\begin{aligned}
p_{R} E & {\left[d_{R}(p)\right]+p_{E} E\left[d_{E}\left(p_{R}, p\right)\right]-c E[(\bar{I}+k)} \\
\wedge & \left.\left(\bar{y}^{*}\left(p_{R}, p\right) \vee I\right)-\bar{I}\right]-E[H((\bar{I}+k) \\
\wedge & \left.\left.\left(\bar{y}^{*}\left(p_{R}, p\right) \vee I\right)-d_{E}\left(p_{R}, p\right)\right)\right] \\
+ & \alpha E\left[\mathrm { G } _ { t + 1 } \left((\bar{I}+k) \wedge\left(\bar{y}^{*}\left(p_{R}, p\right) \vee I\right)\right.\right. \\
& \left.\left.-d_{E}\left(p_{R}, p\right)-d_{R}(p)\right)\right]
\end{aligned}
$$

is jointly concave in $\left(I, p_{R}, p\right)$, where $a \vee b=\max [a, b]$.

Proof. Given $p_{R}$ and $p, p_{R} d_{R}(p)+p_{E} d_{E}\left(p_{R}, p\right)$ is just constant and thus we will not consider them for a while. Since $H(\cdot)$ and $\mathrm{G}_{t+1}(\cdot)$ are concave,

$$
\begin{aligned}
& p_{R} d_{R}(p)+p_{E} d_{E}\left(p_{R}, p\right)-c \bar{y}-H\left(\bar{y}-d_{E}\left(p_{R}, p\right)\right) \\
& \quad+\alpha G_{t+1}\left(\bar{y}-d_{E}\left(p_{R}, p\right)-d_{R}(p)\right)
\end{aligned}
$$

is jointly concave in $\left(\bar{y}, p_{R}, p\right)$. Now, take any $\delta>0$ such that $\bar{y}+\delta<\bar{y}^{*}\left(p_{R}, p\right)$ and we have $\bar{y} \wedge(I+k) \leq(\bar{y}+\delta) \wedge(I+k)<$ $\bar{y}^{*}\left(p_{R}, p\right)$. So,

$$
\begin{aligned}
& -c(\bar{y} \wedge(\bar{I}+k)-\bar{I})-H\left((\bar{I}+k) \wedge \bar{y}-d_{E}\left(p_{R}, p\right)\right) \\
& +\alpha\left(G_{t+1}\left((\bar{I}+k) \wedge \bar{y}-d_{E}\left(p_{R}, p\right)-d_{R}(p)\right)\right) \\
& \leq-c((\bar{y}+\delta) \wedge(\bar{I}+k)-\bar{I})-H((\bar{I}+k) \wedge(\bar{y} \\
& \left.+\delta)-d_{E}\left(p_{R}, p\right)\right)+\alpha\left(\mathrm{G}_{t+1}((\bar{I}+k) \wedge(\bar{y}+\delta)\right. \\
& \left.\left.-d_{E}\left(p_{R}, p\right)-d_{R}(p)\right)\right)
\end{aligned}
$$

for any $k$. Thus,

$$
\begin{aligned}
E & -c(\bar{y} \wedge(\bar{I}+k)-\bar{I})-H((\bar{I}+k) \wedge \bar{y} \\
& \left.-d_{E}\left(p_{R}, p\right)\right)+\alpha \mathrm{G}_{t+1}\left((\bar{I}+k) \wedge \bar{y}-d_{E}\left(p_{R}, p\right)\right. \\
& \left.\left.-d_{R}(p)\right)\right] \leq E[-c((\bar{y}+\delta) \wedge(\bar{I}+k)-\bar{I}) \\
& -H\left((\bar{I}+k) \wedge(\bar{y}+\delta)-d_{E}\left(p_{R}, p\right)\right) \\
& +\alpha \mathrm{G}_{t+1}\left((\bar{I}+k) \wedge(\bar{y}+\delta)-d_{E}\left(p_{R}, p\right)\right. \\
& \left.\left.-d_{R}(p)\right)\right] .
\end{aligned}
$$


Therefore,

$$
\begin{aligned}
& -c E[(\bar{I}+k) \wedge \bar{y}-\bar{I}] \\
& -E\left[H\left((\bar{I}+k) \wedge \bar{y}-d_{E}\left(p_{R}, p\right)\right)\right] \\
& \quad+\alpha E\left[G_{t+1}\left((\bar{I}+k) \wedge \bar{y}-d_{E}\left(p_{R}, p\right)-d_{R}(p)\right)\right]
\end{aligned}
$$

is increasing in $\bar{y}$ when $\bar{y} \leq \bar{y}^{*}\left(p_{R}, p\right)$. By the similar argument,

$$
\begin{aligned}
& -c E[(\bar{I}+k) \wedge \bar{y}-\bar{I}] \\
& -E\left[H\left((\bar{I}+k) \wedge \bar{y}-d_{E}\left(p_{R}, p\right)\right)\right] \\
& \quad+\alpha E\left[G_{t+1}\left((\bar{I}+k) \wedge \bar{y}-d_{E}\left(p_{R}, p\right)-d_{R}(p)\right)\right]
\end{aligned}
$$

is decreasing in $\bar{y}$ when $\bar{y} \geq \bar{y}^{*}\left(p_{R}, p\right)$. The first result holds. Since, for given $p_{R}$ and $p, \bar{y}^{*}\left(p_{R}, p\right)$ is the maximizer of

$$
\begin{gathered}
-c((\bar{I}+k)-\bar{I})-H\left((\bar{I}+k) \wedge \bar{y}-d_{E}\left(p_{R}, p\right)\right) \\
+\alpha \mathrm{G}_{t+1}\left((\bar{I}+k) \wedge \bar{y}-d_{E}\left(p_{R}, p\right)-d_{R}(p)\right)
\end{gathered}
$$

for any $\bar{y} \in \mathbb{R}$, for given $p_{R}$ and $p,\left(\bar{y}^{*}\left(p_{R}, p\right) \vee \bar{I}\right) \wedge(\bar{I}+k)$ is the maximizer of

$$
\begin{gathered}
-c((\bar{I}+k)-\bar{I})-H\left((\bar{I}+k) \wedge \bar{y}-d_{E}\left(p_{R}, p\right)\right) \\
+\alpha \mathrm{G}_{t+1}\left((\bar{I}+k) \wedge \bar{y}-d_{E}\left(p_{R}, p\right)-d_{R}(p)\right)
\end{gathered}
$$

for $\bar{y} \in[\bar{I}, \bar{I}+k]$. Now, take $\left(\bar{I}_{1}, p_{R, 1}, p_{1}\right),\left(\bar{I}_{2}, p_{R, 2}, p_{2}\right)$, and $\gamma \in[0,1]$. Then

$$
\begin{aligned}
\gamma & -c\left(\left(\bar{I}_{1}+k\right) \wedge\left(\bar{y}^{*}\left(p_{R, 1}, p_{1}\right) \vee \bar{I}_{1}\right)-\bar{I}_{1}\right)-H\left(\left(\bar{I}_{1}\right.\right. \\
& \left.+k) \wedge\left(\bar{y}^{*}\left(p_{R, 1}, p_{1}\right) \vee \bar{I}_{1}\right)-d_{E}\left(p_{R, 1}, p_{1}\right)\right) \\
& +\alpha\left(\mathrm { G } _ { t + 1 } \left(\left(\bar{I}_{1}+k\right) \wedge\left(\bar{y}^{*}\left(p_{R, 1}, p_{1}\right) \vee I_{1}\right)\right.\right. \\
& \left.\left.\left.-d_{E}\left(p_{R, 1}, p_{1}\right)-d_{R}\left(p_{R, 1}, p_{1}\right)\right)\right)\right)+(1-\gamma) \\
& \cdot\left(-c\left(\left(\bar{I}_{2}+k\right) \wedge\left(\bar{y}^{*}\left(p_{R, 2}, p_{2}\right) \vee \bar{I}_{2}\right)-\bar{I}_{2}\right)\right. \\
& -H\left(\left(\bar{I}_{2}+k\right) \wedge\left(\bar{y}^{*}\left(p_{R, 2}, p_{2}\right) \vee \bar{I}_{2}\right)\right. \\
& \left.-d_{E}\left(p_{R, 2}, p_{2}\right)\right)+\alpha \mathrm{G}_{t+1}\left(\left(\bar{I}_{2}+k\right)\right. \\
& \wedge\left(\bar{y}^{*}\left(p_{R, 2}, p_{2}\right) \vee \bar{I}_{2}\right)-d_{E}\left(p_{R, 2}, p_{2}\right) \\
& \left.\left.-d_{R}\left(p_{R, 2}, p_{2}\right)\right)\right) \leq-c\left(\gamma \left[\left(\bar{I}_{1}+k\right)\right.\right. \\
& \left.\wedge\left(\bar{y}^{*}\left(p_{R, 1}, p_{1}\right) \vee \bar{I}_{1}\right)\right]+(1-\gamma)\left[\left(\bar{I}_{2}+k\right)\right. \\
& \left.\left.\wedge\left(\bar{y}^{*}\left(p_{R, 2}, p_{2}\right) \vee \bar{I}_{2}\right)\right]-\bar{I}_{\gamma}\right)-H\left(\gamma \left[\left(\bar{I}_{1}+k\right)\right.\right. \\
& \left.\wedge\left(\bar{y}^{*}\left(p_{R, 1}, p_{1}\right) \vee \bar{I}_{1}\right)\right]+(1-\gamma)\left[\left(\bar{I}_{2}+k\right)\right.
\end{aligned}
$$

$$
\begin{aligned}
& \left.\left.\wedge\left(\bar{y}^{*}\left(p_{R, 2}, p_{2}\right) \vee \bar{I}_{2}\right)\right]-d_{E}\left(p_{R, \gamma}, p_{E, \gamma}\right)\right) \\
& +\alpha \mathrm{G}_{t+1}\left(\gamma\left[\left(\bar{I}_{1}+k\right) \wedge\left(\bar{y}^{*}\left(p_{R, 1}, p_{1}\right) \vee \bar{I}_{1}\right)\right]+(1\right. \\
& -\gamma)\left[\left(\bar{I}_{2}+k\right) \wedge\left(\bar{y}^{*}\left(p_{R, 2}, p_{2}\right) \vee \bar{I}_{2}\right)\right] \\
& -\gamma\left[d_{E}\left(p_{R, 1}, p_{1}\right)-d_{R}\left(p_{R, 1}, p_{1}\right)\right]-(1-\gamma) \\
& \left.\cdot\left[d_{E}\left(p_{R, 2}, p_{2}\right)-d_{R}\left(p_{R, 2}, p_{2}\right)\right]\right)=-c\left(\gamma \left[\left(\bar{I}_{1}+k\right)\right.\right. \\
& \left.\wedge\left(\bar{y}^{*}\left(p_{R, 1}, p_{1}\right) \vee \bar{I}_{1}\right)\right]+(1-\gamma)\left[\left(\bar{I}_{2}+k\right)\right. \\
& \left.\left.\wedge\left(\bar{y}^{*}\left(p_{R, 2}, p_{2}\right) \vee \bar{I}_{2}\right)\right]-\bar{I}_{\gamma}\right)-H\left(\gamma \left[\left(\bar{I}_{1}+k\right)\right.\right. \\
& \left.\wedge\left(\bar{y}^{*}\left(p_{R, 1}, p_{1}\right) \vee \bar{I}_{1}\right)\right]+(1-\gamma)\left[\left(\bar{I}_{2}+k\right)\right. \\
& \left.\left.\wedge\left(\bar{y}^{*}\left(p_{R, 2}, p_{2}\right) \vee \bar{I}_{2}\right)\right]-d_{E}\left(p_{R, \gamma}, p_{E, \gamma}\right)\right) \\
& +\alpha \mathrm{G}_{t+1}\left(\gamma\left[\left(\bar{I}_{1}+k\right) \wedge\left(\bar{y}^{*}\left(p_{R, 1}, p_{1}\right) \vee \bar{I}_{1}\right)\right]+(1\right. \\
& -\gamma)\left[\left(\bar{I}_{2}+k\right) \wedge\left(\bar{y}^{*}\left(p_{R, 2}, p_{2}\right) \vee \bar{I}_{2}\right)\right]-d_{E}\left(p_{R, \gamma},\right. \\
& \left.\left.p_{\gamma}\right)-d_{R}\left(p_{R, \gamma}, p_{\gamma}\right)\right) \leq-c\left(\left(\bar{I}_{\gamma}+k\right)\right. \\
& \left.\wedge\left(\bar{y}^{*}\left(p_{R, \gamma}, p_{\gamma}\right) \vee \bar{I}_{\gamma}\right)-\bar{I}_{\gamma}\right)-H\left(\left(\bar{I}_{\gamma}+k\right)\right. \\
& \left.\wedge\left(\bar{y}^{*}\left(p_{R, \gamma}, p_{\gamma}\right) \vee \bar{I}_{\gamma}\right)-d_{E}\left(p_{R, \gamma}, p_{\gamma}\right)\right) \\
& +\alpha \mathrm{G}_{t+1}\left(\left(\bar{I}_{\gamma}+k\right) \wedge\left(\bar{y}^{*}\left(p_{R, \gamma}, p_{\gamma}\right) \vee \bar{I}_{\gamma}\right)\right. \\
& \left.-d_{E}\left(p_{R, \gamma}, p_{\gamma}\right)-d_{R}\left(p_{R, \gamma}, p_{\gamma}\right)\right),
\end{aligned}
$$

where $\bar{I}_{\gamma}=\gamma \bar{I}_{1}+(1-\gamma) \bar{I}_{2}, p_{R, \gamma}=\gamma p_{R, 1}+(1-\gamma) p_{R, 2}$, and $p_{\gamma}=\gamma p_{1}+(1-\gamma) p_{2}$. The first inequality holds due to the concavity of $H_{t}$ and $\mathrm{G}_{t+1}$, the second equality holds due to the linearity of demand functions, and the last inequality holds since, for given $p_{R, \gamma}$ and $p_{\gamma}, \bar{y}=\left(\bar{I}_{\gamma}+k\right) \wedge \bar{y}^{*}\left(p_{R, \gamma}, p_{\gamma}\right) \vee \bar{I}_{\gamma}$ is the maximizer of

$$
\begin{aligned}
& -c(\bar{y}-\bar{I})-H\left(\bar{y}-d_{E}\left(p_{R}, p\right)\right) \\
& \quad+\alpha \mathrm{G}_{t+1}\left(\bar{y}-d_{E}\left(p_{R}, p\right)-d_{R}(p)\right)
\end{aligned}
$$

for $\bar{y} \in\left[\bar{I}_{\gamma}, \bar{I}_{\gamma}+k\right]$. So, we need to verify that

$$
\begin{aligned}
\gamma & {\left[\left(\bar{I}_{1}+k\right) \wedge\left(\bar{y}^{*}\left(p_{R, 1}, p_{1}\right) \vee \bar{I}_{1}\right)\right] } \\
& +(1-\gamma)\left[\left(\bar{I}_{2}+k\right) \wedge\left(\bar{y}^{*}\left(p_{R, 2}, p_{2}\right) \vee \bar{I}_{2}\right)\right]
\end{aligned}
$$

is within $\left[\bar{I}_{\gamma}, \bar{I}_{\gamma}+k\right]$.

$$
\begin{aligned}
\gamma & {\left[\left(\bar{I}_{1}+k\right) \wedge\left(\bar{y}^{*}\left(p_{R, 1}, p_{1}\right) \vee \bar{I}_{1}\right)\right] } \\
& +(1-\gamma)\left[\left(\bar{I}_{2}+k\right) \wedge\left(\bar{y}^{*}\left(p_{R, 2}, p_{2}\right) \vee \bar{I}_{2}\right)\right] \\
& \geq \gamma\left[\left(\bar{I}_{1}+k\right) \wedge \bar{I}_{1}\right]+(1-\gamma)\left[\left(\bar{I}_{2}+k\right) \wedge \bar{I}_{2}\right] \\
& =\gamma \bar{I}_{1}+(1-\gamma) \bar{I}_{2}=\bar{I}_{\gamma},
\end{aligned}
$$


TABLE 1: Parameters for numerical analysis.

\begin{tabular}{ccccccccc}
\hline$a$ & $b_{R}$ & $b_{E}$ & Marginal cost & Holding cost & Backlog cost & Salvage price & Range for regular price $\left(p_{R}\right)$ & Range for extra charge $(p)$ \\
\hline 250 & 5 & 6 & 30 & 0.3 & 28 & 20 & $25-65$ & $0-30$ \\
\hline
\end{tabular}

$$
\begin{aligned}
& \gamma\left[\left(\bar{I}_{1}+k\right) \wedge\left(\bar{y}^{*}\left(p_{R, 1}, p_{1}\right) \vee \bar{I}_{1}\right)\right] \\
& \quad+(1-\gamma)\left[\left(\bar{I}_{2}+k\right) \wedge\left(\bar{y}^{*}\left(p_{R, 2}, p_{2}\right) \vee \bar{I}_{2}\right)\right] \\
& \quad \leq \gamma\left(\bar{I}_{1}+k\right)+(1-\gamma)\left(\bar{I}_{2}+k\right)=\bar{I}_{\gamma}+k .
\end{aligned}
$$

By taking the expectation, we can obtain the second result.

Proposition 9. For all $t=1,2, \ldots, T, \mathrm{G}_{t}(\cdot)$ is concave function.

Proof. Since we consider the $T$-period problem, $\mathrm{G}_{T+1}(\bar{I})=$ 0 and $\mathrm{G}_{T+1}(\bar{I})$ is concave in $\bar{I}$. By induction, suppose that $\mathrm{G}_{t+1}(\cdot)$ is concave. Let $\bar{y}^{*}\left(p_{R}, p\right)$ be defined as in Lemma 8. By Assumption 4 and Lemma 8,

$$
\begin{aligned}
\Pi_{t} & \left(\bar{I}: \bar{y}^{*}\left(p_{R}, p\right) \vee \bar{I}, p_{R}, p\right)=p_{R} E\left[d_{R}(p)\right] \\
& +p_{E} E\left[d_{E}\left(p_{R}, p\right)\right]-c E\left[( \overline { I } + k ) \wedge \left(\bar{y}^{*}\left(p_{R}, p\right)\right.\right. \\
& \vee \bar{I})-\bar{I}]-E\left[H \left((\bar{I}+k) \wedge\left(\bar{y}^{*}\left(p_{R}, p\right) \vee \bar{I}\right)\right.\right. \\
& \left.\left.-d_{E}\left(p_{R}, p\right)\right)\right]+\alpha E\left[G_{t+1}((\bar{I}+k)\right. \\
& \left.\left.\wedge\left(\bar{y}^{*}\left(p_{R}, p\right) \vee \bar{I}\right)-d_{E}\left(p_{R}, p\right)-d_{R}(p)\right)\right]
\end{aligned}
$$

is jointly concave in $\left(\bar{I}, p_{R}, p\right)$. So,

$$
\mathrm{G}_{t}(\bar{I})=\max _{\underline{p}_{R} \leq p_{R} \leq \bar{p}_{R}, \underline{p} \leq p \leq \bar{p}} \Pi_{t}\left(\bar{I}: \bar{y}^{*}\left(p_{R}, p\right) \vee \bar{I}, p_{R}, p\right)
$$

is concave in $\bar{I}$. Therefore, for all $t=1,2, \ldots, T, \mathrm{G}_{t}(\cdot)$ is concave.

Proposition 10. Suppose that, in period $t$, the inventory level is $I_{t}$ and the advanced demand from period $t-1$ which is for regular service is $x_{t-1}$. Then, in period $t$, there is some finite pair $\left(y_{t}^{*}, p_{t}^{*}, p_{R, t}^{*}\right)$ such that if $I_{t}-x_{t-1} \leq y_{t}^{*}$, it is optimal to place positive number $\left(y_{t}^{*}-I_{t}+x_{t-1}\right)$ of order and to charge the prices $p_{R, t}^{*}+p_{t}^{*}$ for the express service and $p_{R, t}^{*}$ for the regular service. Otherwise, no ordering will take place.

Proof. As seen in Lemma 8, the optimal solution pair $\left(y_{t}^{*}, p_{R, t}^{*}, p_{t}^{*}\right)$ does not depend on $I_{t}$ and $x_{t-1}$ but $y_{t}^{*}$ depends on $\left(p_{R}^{*}, p_{t}\right)$ which is $y_{t}^{*}=\bar{y}_{t}^{*}\left(p_{R, t}^{*}, p_{t}^{*}\right)$. So, if $I_{t}-x_{t-1} \leq y_{t}^{*}$, it is optimal to place $y_{t}^{*}-I_{t}+x_{t-1}$ of orders. Otherwise, the optimal order up to level is $I_{t}-x_{t-1}$ which is not to order. Now, we need to verify that there exists finite solution pair in each period $t$. In each period $t$, the feasible sets for $p_{R, t}$ and $p_{t}$ are bounded so that the optimal pricing $p_{R, t}^{*}$ and $p_{t}^{*}$ are finite. $y_{t}^{*}=\bar{y}_{t}^{*}\left(p_{R, t}^{*}, p_{t}^{*}\right)$ is solution to

$$
\begin{aligned}
& p_{R, t}^{*} d_{R}\left(p_{t}^{*}\right)+\left(p_{R, t}^{*}+p_{t}^{*}\right) d_{E}\left(p_{R, t}^{*}, p_{t}^{*}\right)-c(\bar{y}-\bar{I}) \\
& \quad-H\left(\bar{y}-d_{E}\left(p_{R, t}^{*}, p_{t}^{*}\right)\right) \\
& \quad+\alpha \mathrm{G}_{t+1}\left(\bar{y}-d_{E}\left(p_{R, t}^{*}, p_{t}^{*}\right)-d_{R}\left(p_{t}^{*}\right)\right) .
\end{aligned}
$$

By Assumption 6, in each period $t$,

$$
\lim _{\bar{y} \rightarrow \pm \infty}-c \bar{y}-H\left(\bar{y}-d_{E}\left(p_{R, t}^{*}, p_{t}^{*}\right)\right)=-\infty .
$$

Thus, as $\bar{y} \rightarrow \pm \infty$

$$
\begin{aligned}
& \left(p_{R, t}^{*} d_{R}\left(p_{t}^{*}\right)+\left(p_{R, t}^{*}+p_{t}^{*}\right) d_{E}\left(p_{R, t}^{*}, p_{t}^{*}\right)-c(\bar{y}-\bar{I})\right. \\
& \left.\quad-H\left(\bar{y}-d_{E}\left(p_{R, t}^{*}, p_{t}^{*}\right)\right)\right)+\alpha \mathrm{G}_{t+1}(\bar{y} \\
& \left.\quad-d_{E}\left(p_{R, t}^{*}, p_{t}^{*}\right)-d_{R}\left(p_{t}^{*}\right)\right)
\end{aligned}
$$

will go to $\infty$, and thus the solution $y_{t}^{*}=\bar{y}_{t}^{*}\left(p_{R, t}^{*}, p_{t}^{*}\right)$ should be finite. Therefore, the result holds.

\section{Numerical Analysis}

In this section, we provide a report on a numerical analysis carried on to obtain insights into the structure of optimal policies and their sensitivity and quantitative comparison with the traditional policy (single-pricing policy). Among the main questions, we focus on

(1) the benefits of a multiple pricing strategy compared to a one pricing strategy in settings with continuous inventory replenishment opportunities,

(2) the sensitivity of the optimal base stock and list prices with respect to the degree of variability and the seasonality in the demands,

(3) the comparison of profit with the traditional singlepricing policy.

Our numerical study is based on data in Table 1. As mentioned in Assumption 3, the demand function is a linear function of the regular price and extra charge. That is,

$$
d_{E}\left(p_{r}, p\right)=a \times \gamma-b_{R} p_{R}-b_{E} p+\epsilon .
$$

The random term $\epsilon$ is assumed to be normally distributed with mean 0 . However, it is truncated to avoid the negative value of demand such that the minimum of $\epsilon$ is $-1 \times(a-$ $\left.b_{R} p_{R}-b_{E} p\right)$. Also, to capture the degree of demand variability, we have used [c.v. $\left.\times\left(a-b_{R} p_{R}-b_{E} p\right)\right]^{2}$ as the variance of $\epsilon$, 


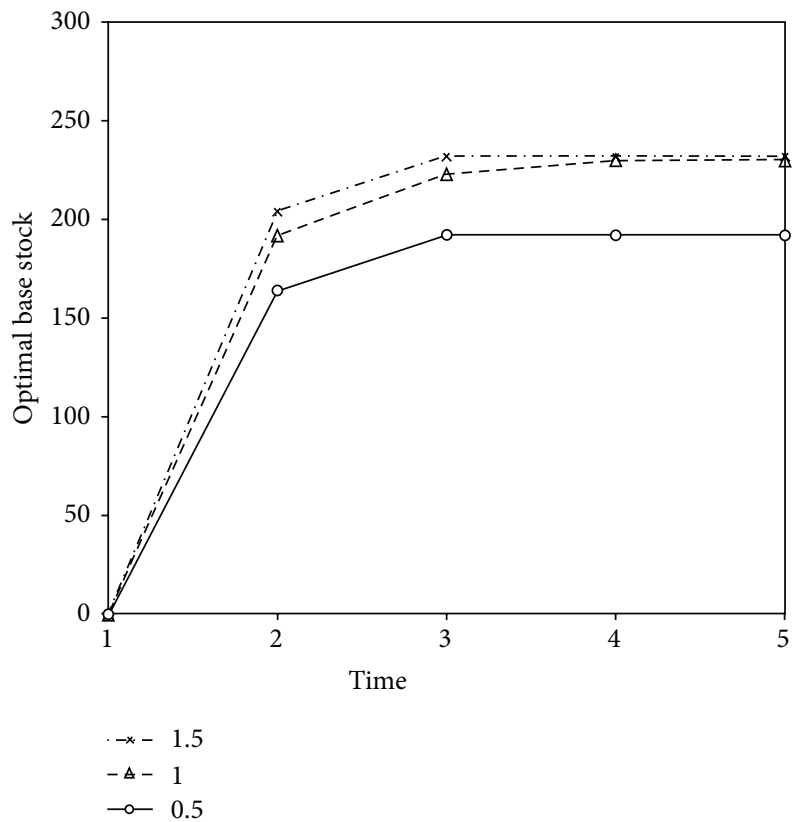

(a) Nonseasonal case

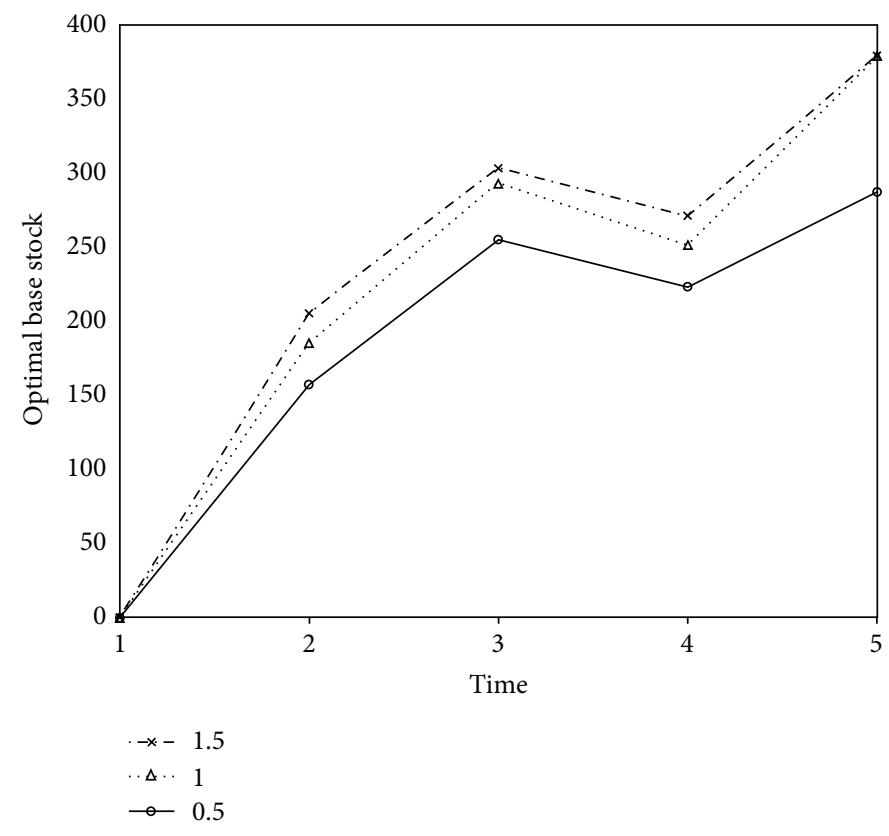

(b) Seasonal case

FIGURE 1: Optimal base stock levels for varying demand uncertainty.

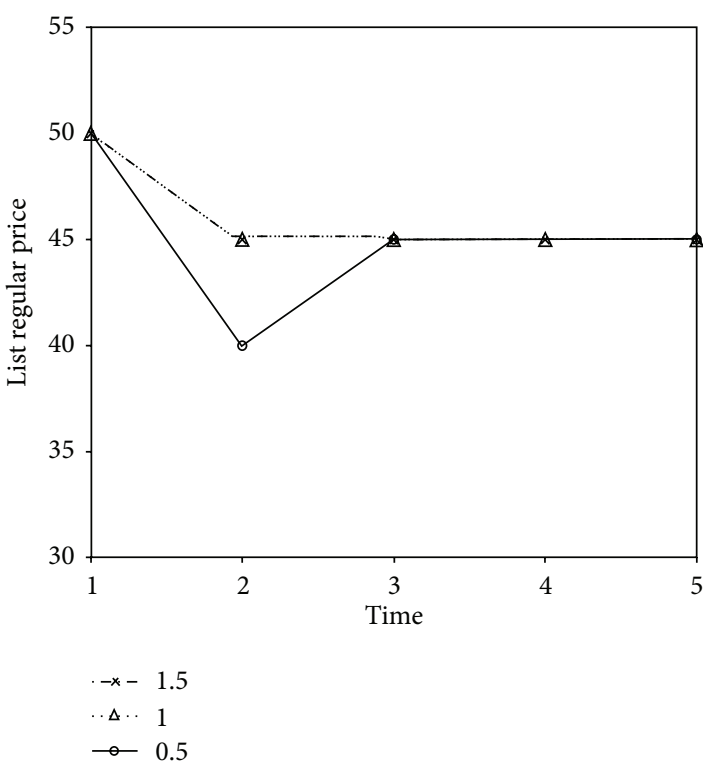

(a) Nonseasonal case

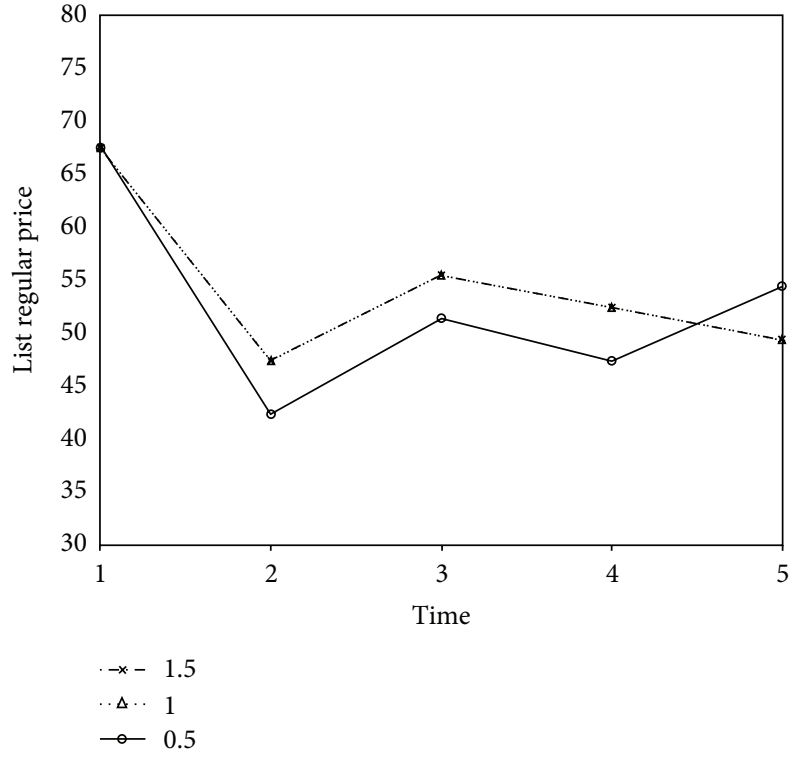

(b) Seasonal case

FIGURE 2: Price for regular service for varying demand uncertainty.

where c.v. is the coefficient for the variability in demand. $\gamma$ is the randomly generated number for the demand seasonality.

Figure 1 shows the base stock for both nonseasonal and seasonal demand cases. As you can see in this figure, as the demand variation increases, the base stock tends to increase. This might be from the fact that more demand fluctuation can increase the possibility of inventory shortage. So, in order to decrease the shortage cost, the base stock would increase. Figures 2 and 3 show the threshold price predetermined for regular service and express service in each time for each demand variation (c.v.).

The inventory controlling strategy by multiple service levels can provide the retailer or producer with the oneperiod advanced information regarding demand since some customers, who select regular service, are willing to delay shipment for their order. This one-period advanced information can be useful in managing the inventory in the next period in the sense that inventory can be controlled 


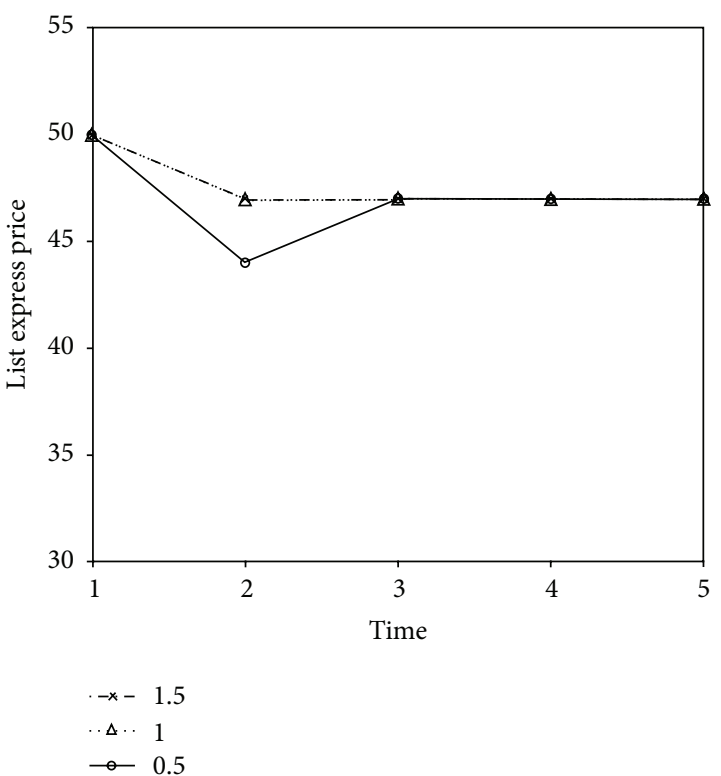

(a) Nonseasonal case

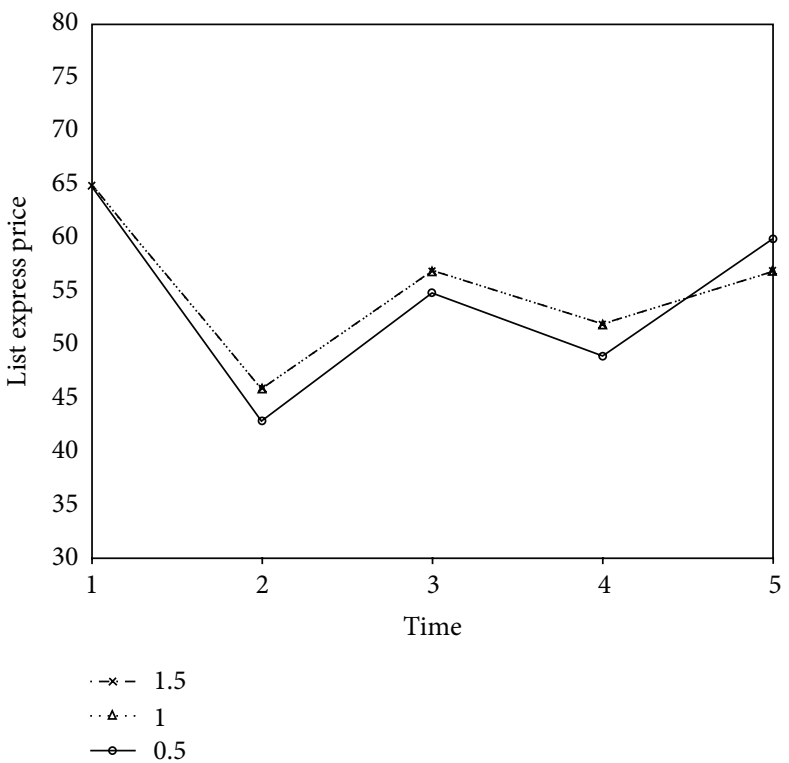

(b) Seasonal case

FIGURE 3: Price for express service for varying demand uncertainty.

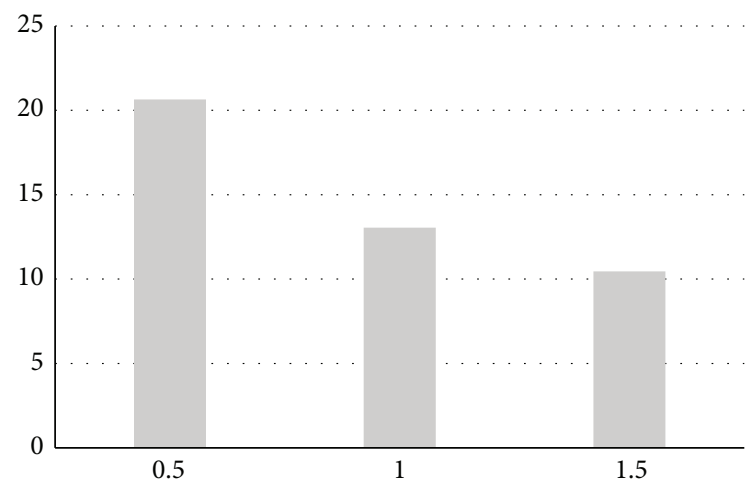

(a) Nonseasonal case

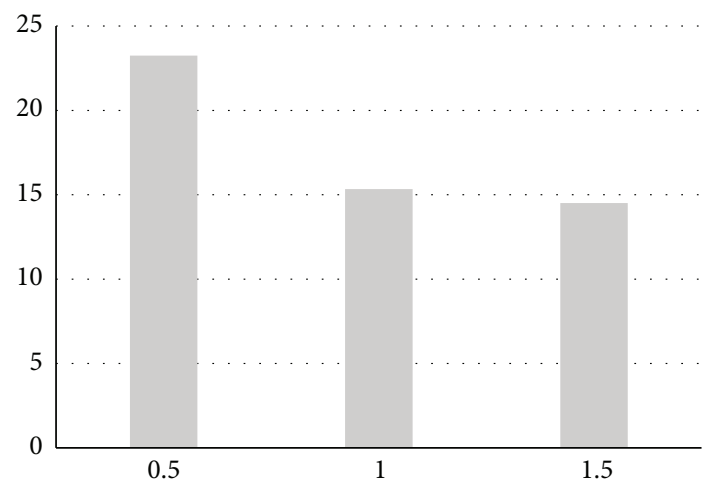

(b) Seasonal case

FIgURE 4: Profit comparison between inventory control by multiple service levels and traditional inventory control by single-service level.

more efficiently and moreover there is chance to reduce the cost which is induced from holding too much inventory or shortage inventory.

Figure 4 shows the benefits of an inventory control through multiple service levels compared to traditional inventory control by single-service level. In both nonseasonal and seasonal case, you can see that our model under unreliable supplying capacity is more profitable than the traditional single-service and single-pricing model. Moreover, at higher level of demand uncertainty (c.v.), the profit increase from our model for seasonal demand case is higher than the nonseasonal case. Thus, we can see that the benefit from inventory control strategy by multiple service levels can be relatively large in the environment where the inventory system experiences higher demand uncertainty and seasonality: inventory control by multiple service levels under the unreliable supplying capacity, which provides the system with one-period advanced information regarding demand, efficiently captures the demand uncertainty in order to reduce the cost compared to traditional controlling model by singleservice level, thus giving more profit. From this numerical analysis, we can see that the proposed model provides better strategy for the inventory controlling problem even under the unreliable supplying capacity.

\section{Conclusion}

We study dynamic pricing and inventory replenishment problems under unreliable supplying condition. This research was initiated by the following practical intuition; that is, if customers are willing to expedite the service for their order, then they are willing to pay extra charge. In this paper, we have verified this intuition through constructing a reasonable demand model (Assumption 3) and by the 
mathematical dynamic programming model where product can be provided to the customers with multiple (two) prices corresponding to the service level, either express service or regular service. It is shown in this paper that there exist a pair of threshold levels for inventory ordering up to level and prices corresponding to service levels in the following strategy: if the inventory level before ordering in each period which is subtracted by the regular demand advanced from the previous period is less than the predetermined threshold, then it is optimal to make ordering decision and increase inventory level up to the predetermined threshold level and offer threshold price for each service level. Otherwise, no ordering is optimal.

We can extend our model in the following possible research directions. First, we can extend the result to the case of infinite horizon. Considering an infinite horizon model with stationary parameters with some practical assumptions, some optimality result similar to one from the finite horizon model might be extended. Second, we assume the dependency of demand in a period only on the price in the same and current period. However, the demand function might be extended to depend not only on the current period but also on the current and the historical prices, which are the prices in past periods. Even though it would be complex to analyze, it would be interesting to incorporate these more general demand settings into the model and examine the corresponding optimal policies. Third, our assumption in which the backlogging is allowed can be modified such that the backlogging is not allowed but lost-sales are assumed. Finally, it would be interesting and important to incorporate a fixed ordering cost into our model. Analysis of such extensions can be more complicated to analyze but would be worth further exploration.

\section{Competing Interests}

The authors declare that there are no competing interests regarding the publication of this paper.

\section{Acknowledgments}

This research was supported by 2014 Hongik University Research Fund and also partially supported by the National Research Foundation of Korea Grant funded by the Korean Government (NRF-2015R1C1A1A02037096). These research funds do not lead to any competing interests regarding the publication of this manuscript.

\section{References}

[1] W. Elmaghraby and P. Keskinocak, "Dynamic pricing in the presence of inventory considerations: research overview, current practices, and future directions," Management Science, vol. 49, no. 10, pp. 1287-1309, 2003.

[2] Q. Feng, "Integrating dynamic pricing and replenishment decisions under supply capacity uncertainty," Management Science, vol. 56, no. 12, pp. 2154-2172, 2010.

[3] S. Karlin and C. R. Carr, "Prices and optimal inventory policy," in Studies in Applied Probability and Management Science, vol. 4, pp. 159-172, 1962.
[4] N. C. Petruzzi and M. Dada, "Pricing and the newsvendor problem: a review with extensions," Operations Research, vol. 47, no. 2, pp. 183-194, 1999.

[5] V. Agrawal and S. Seshadri, "Impact of uncertainty and risk aversion on price and order quantity in the newsvendor problem," Manufacturing and Service Operations Management, vol. 2, no. 4, pp. 410-423, 2000.

[6] A. Federgruen and A. Heching, "Combined pricing and inventory control under uncertainty," Operations Research, vol. 47, no. 3, pp. 454-475, 1999.

[7] J. Lee, "Inventory control by different service levels," Applied Mathematical Modelling, vol. 35, no. 1, pp. 497-505, 2011.

[8] X. Chen and D. Simchi-Levi, "Coordinating inventory control and pricing strategies with random demand and fixed ordering cost: the finite horizon case," Operations Research, vol. 52, no. 6, pp. 887-896, 2004.

[9] X. Chen and D. Simchi-Levi, "Coordinating inventory control and pricing strategies with random demand and fixed ordering cost: the infinite horizon case," Mathematics of Operations Research, vol. 29, no. 3, pp. 698-723, 2004.

[10] J. Lee, "Dynamic pricing inventory control under fixed cost and lost sales," Applied Mathematical Modelling, vol. 38, no. 2, pp. 712-721, 2014.

[11] M. Güray Güler, T. Bilgiç, and R. Güllü, "Joint pricing and inventory control for additive demand models with reference effects," Annals of Operations Research, vol. 226, no. 1, pp. 255276, 2015.

[12] S. Li, J. Zhang, and W. Tang, "Joint dynamic pricing and inventory control policy for a stochastic inventory system with perishable products," International Journal of Production Research, vol. 53, no. 10, pp. 2937-2950, 2015.

[13] Y. Chen and C. Shi, "Joint pricing and inventory management with strategic customers," 2016.

[14] L. Feng, J. Zhang, and W. Tang, "Optimal inventory control and pricing of perishable items without shortages," IEEE Transactions on Automation Science and Engineering, vol. 13, no. 2, pp. 918-931, 2016.

[15] C. A. Yano and H. L. Lee, "Lot sizing with random yields: a review," Operations Research, vol. 43, no. 2, pp. 311-334, 1995.

[16] Y. Wang and Y. Gerchak, "Periodic review production models with variable capacity, random yield, and uncertain demand," Management Science, vol. 42, no. 1, pp. 130-137, 1996.

[17] Q. Li and S. Zheng, "Joint inventory replenishment and pricing control for systems with uncertain yield and demand," Operations Research, vol. 54, no. 4, pp. 696-705, 2006.

[18] X. Chao, H. Chen, and S. Zheng, "Joint replenishment and pricing decisions in inventory systems with stochastically dependent supply capacity," European Journal of Operational Research, vol. 191, no. 1, pp. 142-155, 2008.

[19] P. Ignaciuk, "Discrete-time control of production-inventory systems with deteriorating stock and unreliable supplies," IEEE Transactions on Systems, Man, and Cybernetics: Systems, vol. 45, no. 2, pp. 338-348, 2015.

[20] L. Shu, F. Wu, J. Ni, and L. K. Chu, "On the risk-averse procurement strategy under unreliable supply," Computers \& Industrial Engineering, vol. 84, pp. 113-121, 2015.

[21] B. Hu and D. Kostamis, "Managing supply disruptions when sourcing from reliable and unreliable suppliers," Production and Operations Management, vol. 24, no. 5, pp. 808-820, 2015.

[22] K. Park and K. Lee, "Distribution-robust single-period inventory control problem with multiple unreliable suppliers," $O R$ Spectrum, 2016. 
[23] F. Chen, "Market segmentation, advanced demand information, and supply chain performance," Manufacturing \& Service Operations Management, vol. 3, no. 1, pp. 53-67, 2001.

[24] L. Li, "A stochastic theory of the firm," Mathematics of Operations Research, vol. 13, no. 3, pp. 447-466, 1988. 


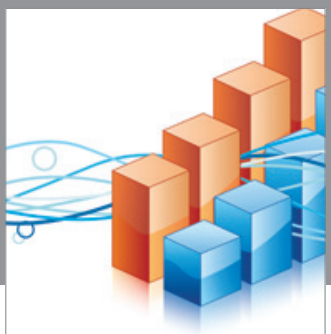

Advances in

Operations Research

vatem alat4

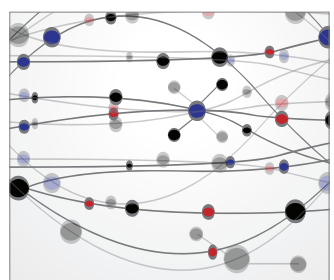

\section{The Scientific} World Journal
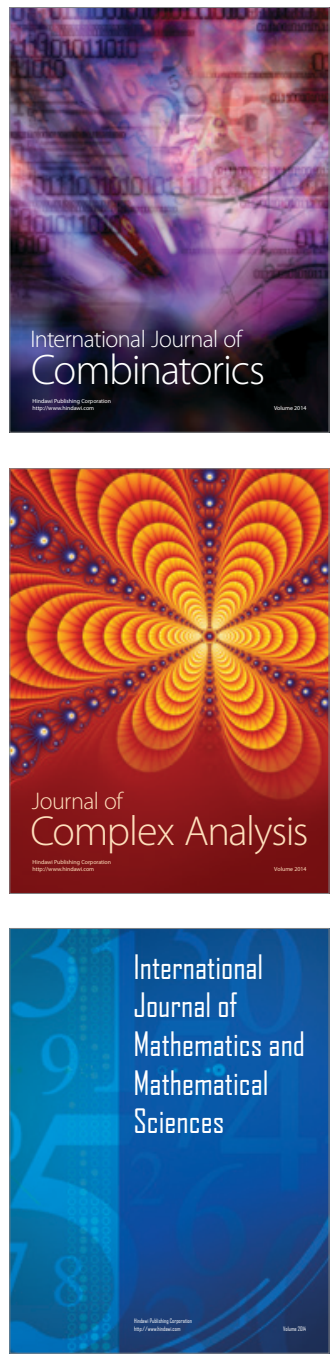
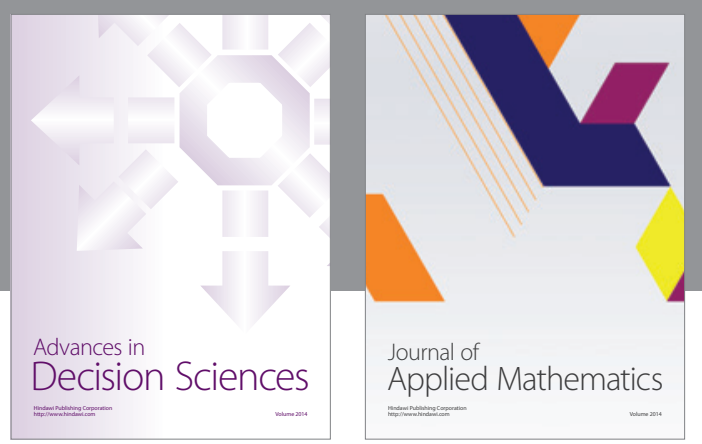

Algebra

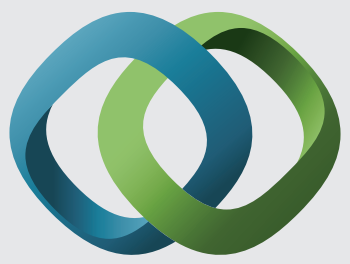

\section{Hindawi}

Submit your manuscripts at

http://www.hindawi.com
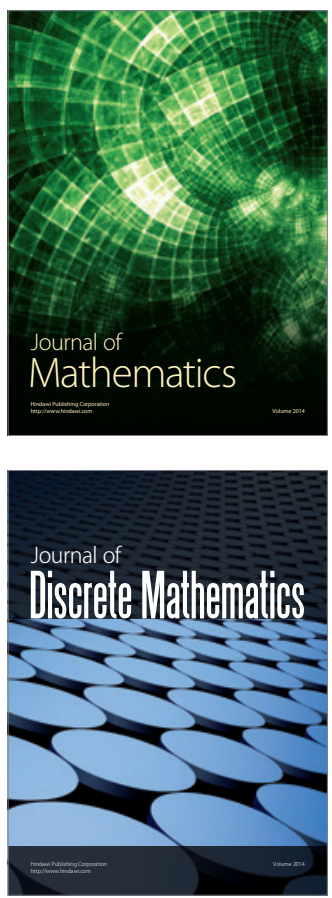

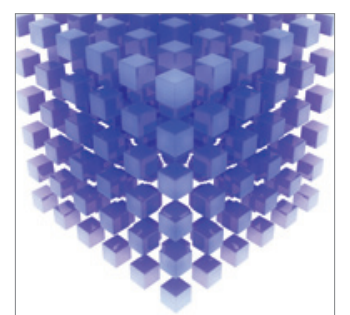

Mathematical Problems in Engineering
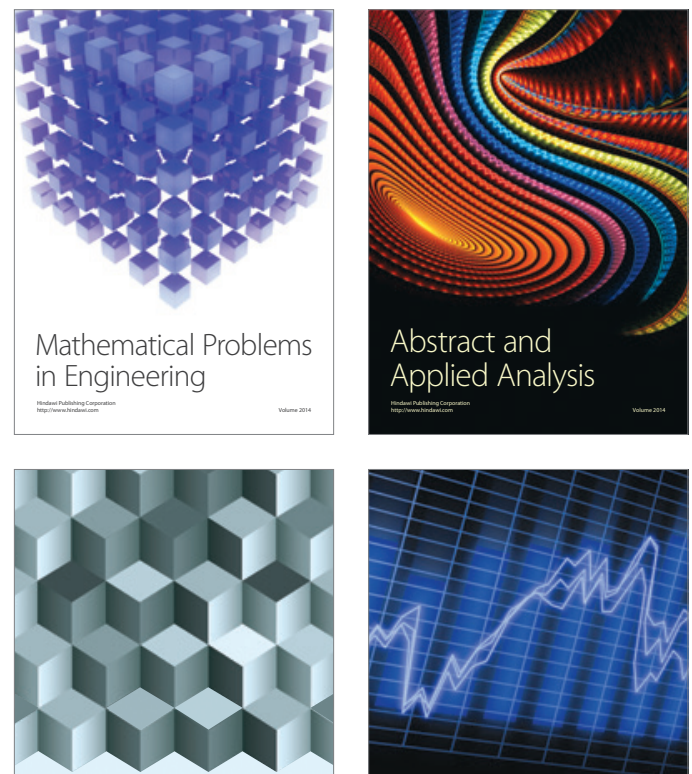

Journal of

Function Spaces

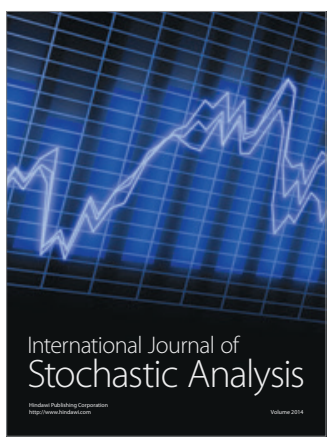

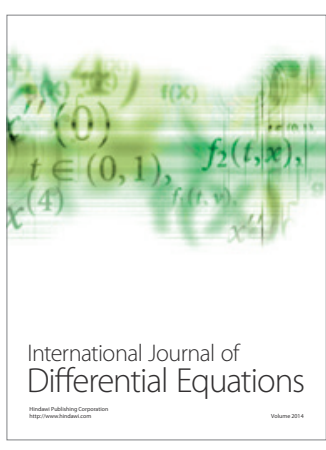
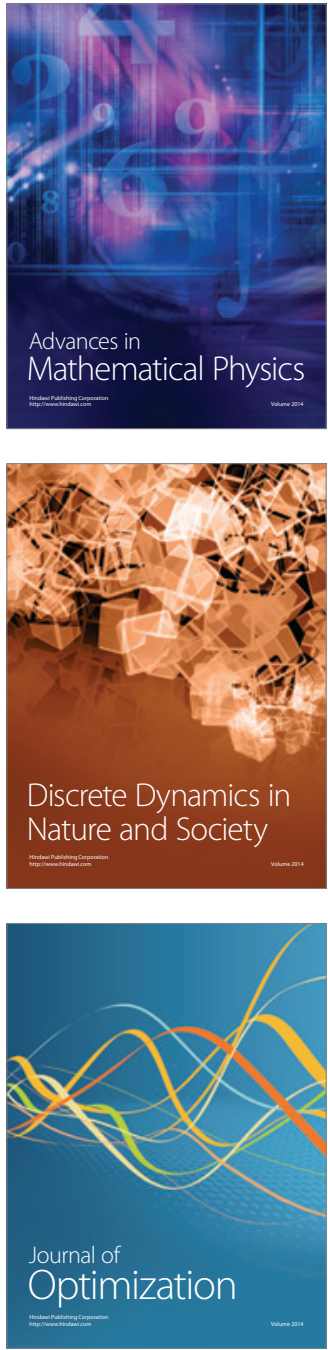